MENDES, R.S., SOUZA, A.P. e SANTANA, V.L. Tetralogia de Fallot em pequenos animais -

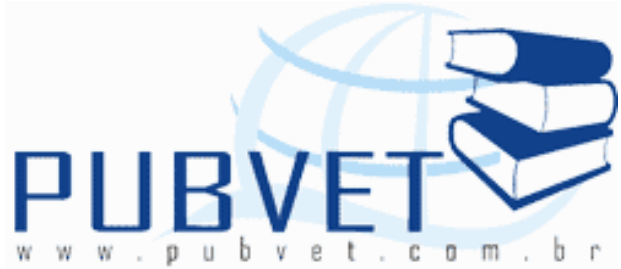

PUBVET, Publicações em Medicina Veterinária e Zootecnia.

\title{
Tetralogia de Fallot em pequenos animais - Revisão bibliográfica
}

Rodrigo de Souza Mendes ${ }^{1}$, Almir Pereira de Souza ${ }^{2}$, Vanessa Lira de Santana ${ }^{3}$

${ }^{1}$ Médico Veterinário, Mestrando, Programa de Pós-graduação em Medicina Veterinária, Universidade Federal de Campina Grande, Campus de Patos, PB. Email: rodrigoexalta@hotmail.com

${ }^{2}$ Médico Veterinário, Professor, Doutor, Universidade Federal de Campina Grande, Campus de Patos, PB. Email: almir@ufcg.csrt.edu.br

${ }^{3}$ Médica Veterinária, Mestre, Universidade Federal de Campina Grande, Campus de Patos, PB. Email: nessavetufcg@yahoo.com.br

\section{Resumo}

A Tetralogia de Fallot (TF) é uma cardiopatia congênita causadora de cianose, reconhecida, classicamente, como a combinação de quatro defeitos. Sua fisiopatologia está intimamente relacionada com dois defeitos que interferem significantemente na hemodinâmica do animal: a estenose da valva pulmonar e o defeito septal interventricular, sendo que suas conseqüências dependem sensivelmente da magnitude destes defeitos. Atualmente, existem vários métodos de diagnóstico, capazes de elucidar com precisão essa cardiopatia congênita. Existem varias modalidades de tratamento, que vão desde terapia medicamentosa a procedimento cirúrgico corretivo, sendo que, atualmente a técnica mais empregada é o método cirúrgico paliativo de Blalock-Taussig.

Palavras-chave: Tetralogia de Fallot, cardiopatia congênita, Blalock-Taussig 
MENDES, R.S., SOUZA, A.P. e SANTANA, V.L. Tetralogia de Fallot em pequenos animais Revisão bibliográfica. PUBVET, Londrina, V. 6, N. 3, Ed. 190, Art. 1279, 2012.

\section{Tetralogy of Fallot in small animals - Bibliographic review}

\section{Abstract}

Tetralogy of Fallot (TF) is a congenital heart defect that causes cyanosis, known classically as a combination of four defects. Its pathophysiology is closely related to two defects that interfere significantly in the hemodynamics of the animal: a pulmonary valve stenosis and ventricular septal defect, and its consequences depend sensitively on the magnitude of these defects. Currently, there are several diagnostic tests capable of accurately elucidate this congenital heart disease. There are several treatment modalities, ranging from medical therapy to surgical correction. However, currently the most widely used surgical method is the palliative Blalock-Taussig shunt.

Keywords: Tetraloy of Fallot, congenital heart disease, Blalock-taussig

\section{INTRODUÇÃO}

A compreensão das alterações fisiopatológicas que acompanham os defeitos cardíacos congênitos é um importante aspecto para seu diagnóstico e tratamento. Visto que o diagnóstico nem sempre é direto, uma apreciação das alterações fisiológicas exibidas por um paciente enfermo poderá ser útil na avaliação dos sintomas clínicos, achados físicos e resultados de outras práticas diagnosticas. A história natural de determinados defeitos cardíacos congênitos é fator importante na compreensão de como um determinado animal pode responder a um distúrbio específico (ANDERSON, 1996).

A cardiopatia congênita constitui a causa mais comum de cardiovasculopatias nos animais com menos de um ano de idade. Dentre essas temos a cardiopatia causadora de cianose mais comum dentre as doenças cardíacas dos animais domésticos, a Tetralogia de Fallot (TF) (MILLER \& BONAGURA, 1998; BONAGURA \& LEHMKHL, 1998). 
MENDES, R.S., SOUZA, A.P. e SANTANA, V.L. Tetralogia de Fallot em pequenos animais Revisão bibliográfica. PUBVET, Londrina, V. 6, N. 3, Ed. 190, Art. 1279, 2012.

Objetivou-se com o presente trabalho apresentar as características gerais da cardiopatia congênita Tetralogia de Fallot em pequenos animais, abordando desde sua fisiopatologia a suas condutas terapêuticas.

\section{TETRALOGIA DE FALLOT (TF)}

A Tetralogia de Fallot (TF) é uma cardiopatia congênita que manifesta cianose, reconhecida, classicamente, como a combinação de quatro defeitos: comunicação interventricular (defeito de septo interventricular), dextroposição da artéria aorta, obstrução da via de saída do ventrículo direito (estenose pulmonar) e hipertrofia congênita secundária do ventrículo direito (Figura 1). Já foi descrito um caso isolado de pentalogia de Fallot num Poodle Toy com um ano de idade, onde o mesmo manifestava TF + Persistência de Ducto Arterioso (PDA) (SISSON et al., 1992; BOON, 1998; BELERENIAN, 2003).

É considerada dentre as moléstias cardíacas congênitas cianóticas a de maior ocorrência. A cianose decorre do desvio de fluxo sanguíneo através do defeito septal interventricular e pela estenose da valva pulmonar (BONAGURA, 1992; EYSTER, 1993; LARSSON et al., 2000).

Para tal afecção, nota-se predisposição racial em cães, acometendo freqüentemente Bulldog Inglês, Keeshond, Poodles, Schnauzers, Fox Terrier, Collie e Pastor de Shetland. Notou-se efeito poligênico em cães da raça Keeshound (BONAGURA, 1992; LARSSON et al., 2000, GOODWIN, 2002). Em gatos, apensar de incomum, sua presença também já foi reconhecida e, dentre as malformações congênitas causadoras de cianose, esta é a mais encontrada (BOLTON et al., 1972; ABBOT, 2000). 

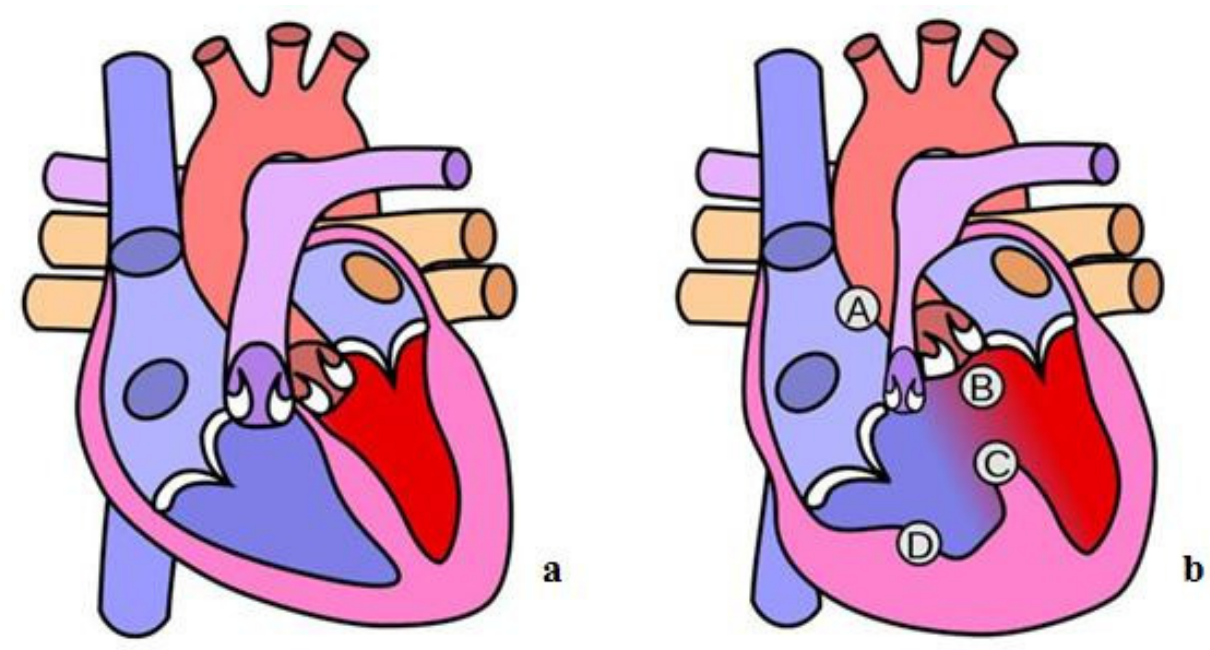

Figura 1: a. Esquema representativo de um coração normal. b. Coração com Tetralogia de Fallot. Demonstrando os quatro defeitos cardíacos (A, B, C, D) encontrados nesta cardiopatia congênita (BARTELINGS \& GITTENBERGER-de GROOT, 1991).

Com o advento da ecocardiografia e angiografia, atualmente, é possível assegurar, um diagnóstico preciso, refletido na determinação de um maior numero de casos (LARSSON et al., 2000).

Estudos embriológicos e hereditários realizados com cães da raça Keehounds, observou-se que a TF faz parte de uma série de manifestações fenotípicas possíveis, decorrentes de uma única anomalia: a malformação do septo conotruncal, na fase de diferenciação de estruturas circulatórias e cavidades cardíacas fetais. Nessas condições, o septo conotruncal forma-se mais cranialmente que o normal, a partir da porção superior do septo interventricular, resultando em mau alinhamento em relação à região inferior do mesmo (PATTERSON et al., 1974; PATTERSON et al., 1993).

Assim, os defeitos conotruncais apresentam-se em diferentes graus de lesões, que variam de assintomáticas a clinicamente complicadas e imcompativeis com a vida, como se segue. Grau 1: persistência do septo do cônus, aneurisma do septo interventricular e ausência do músculo do cônus (curso assintomático e difícil reconhecimento ecocardiográfico). Grau2: lesões 
MENDES, R.S., SOUZA, A.P. e SANTANA, V.L. Tetralogia de Fallot em pequenos animais Revisão bibliográfica. PUBVET, Londrina, V. 6, N. 3, Ed. 190, Art. 1279, 2012.

de grau 1 mais estenose pulmonar ou comunicação interventricular. Grau 3: tetralogia de fallot mais grau 1 de lesões. Alguns podem apresentar a forma extrema, com atresia da artéria pulmonar. Ainda, a aorta pode ser dilatada e tortuosa e haver anomalias do sistema do arco aórtico (SISSON et al., 1992).

\section{Fisiopatologia}

A fisiopatologia da Tetralogia de Fallot está intimamente relacionada com dois defeitos que interferem significantemente na hemodinâmica do animal: a estenose da valva pulmonar e o defeito septal interventricular, sendo que suas conseqüências dependem sensivelmente da magnitude destes defeitos (FOSSUM, 1997; LEW et al., 1998).

No caso em que o defeito septal interventricular está presente com uma estenose de valva pulmonar insignificante, ter-se-á como resultado um desvio no fluxo sanguíneo pelo defeito do ventrículo esquerdo para o direito, levando ao quadro de insuficiência cardíaca esquerda. Vale ressaltar que, nestes casos, o animal apresenta-se acianótico já que a circulação pulmonar não interfere na sistêmica (FOSSUM, 1997; LEW et al., 1998; LARSSON et al., 2000).

Para os casos em que a estenose da valva pulmonar é mais pronunciada, tem-se um desvio de sangue pelo defeito septal interventricular, do ventrículo direito para o esquerdo, provocado pelo aumento da pressão interna do ventrículo direito decorrente de estenose. Com isso, observa-se que o sangue ejetado pela aorta é proveniente principalmente da circulação arterial pulmonar, porém previamente à hematose, o que resultará em severa hipoxemia, diminuição da saturação de oxigênio pela hemoglobina, cianose e policitemia secundária ao aumento da concentração plasmática da eritropoietina (ETTINGER \& SUTER, 1970; BONAGURA, 1992; EYSTER, 1993; LEW et al., 1998; LARSSON et al., 2000).

A hipertrofia ventricular direita é decorrente da estenose da valva pulmonar, devido à maior resistência que esta proporciona a ejeção de sangue pelo ventrículo direito (ETTINGER \& SUTER, 1970). 
MENDES, R.S., SOUZA, A.P. e SANTANA, V.L. Tetralogia de Fallot em pequenos animais Revisão bibliográfica. PUBVET, Londrina, V. 6, N. 3, Ed. 190, Art. 1279, 2012.

Devido ao baixo fluxo sanguíneo pulmonar, as artérias brônquicas dilatam-se para compensar esse déficit e passam a ser denominadas artérias colaterais aortopulmonares, sendo responsáveis pela formação de varicosidades por todo o mediastino e parede torácica (KOBAYASHI et al., 1991).

Dentre os principais efeitos provocados pela hipoxemia está o sensível aumento nas concentrações plasmáticas de eritropoetina, culminando em aumento no hematócrito, chegando a picos de 65 a 68\%, gerando o quadro de policitemia secundária. Tal episódio pode predispor ao aparecimento de trombose e outras complicações vasculares, bem como a acidose metabólica (BONAGURA, 1992).

\section{Sinais clínicos}

Os principais achados clínicos estão relacionados com o quadro de hipoxemia com cianose evidente, nem sempre observada em outras cardiopatias, provocado pelo desvio de sangue da circulação pulmonar para a sistêmica pelo defeito septal ventricular, no qual se verifica retardo no crescimento, intolerância a exercício, fraqueza, dispnéia, ansiedade, síncopes e convulsões, este ultimo em decorrência da policitemia (BONAGURA, 1992; EYSTER, 1993; LARSSON et al., 2000; ABBOT, 2000; KITTLERSON, 1998; BELERENIAN, 2003). O animal pode ser assintomático ou apresentar um tamanho menor em relação aos outros filhotes da ninhada (BELERENIAN, 2003).

Ao exame físico, nota-se à auscultação murmúrio sistólico do tipo ejeção originado pela estenose pulmonar. O que pode ocorrer, no entanto, é a não verificação de tal murmúrio, decorrente principalmente da hiperviscosidade do sangue pela policitemia, o que diminui o som da turbulência ou quando ocorre um equilíbrio de pressão entre o ventrículo direito e esquerdo (ETTINGER \& SUTER, 1970; BONAGURA, 1992; LARSSON et al., 2000). De modo geral, é observado sopro sistólico basilar esquerdo, que pode estar evidente também 
MENDES, R.S., SOUZA, A.P. e SANTANA, V.L. Tetralogia de Fallot em pequenos animais Revisão bibliográfica. PUBVET, Londrina, V. 6, N. 3, Ed. 190, Art. 1279, 2012.

sobre o hemitórax direito, na forma de um sopro sistólico suave, associado ao defeito septal ventricular, embora esse achado seja variável, e a intensidade atenuar-se na presença de policitemia grave. Na palpação, uma vibração pode ser percebida no terceiro espaço intercostal esquerdo, próximo a junção costocondral (WARE, 2003; WARE, 2007; GOODWIN, 2002).

O pulso femoral costuma ser normal e a cianose nos animais acometidos não melhora de maneira apreciável com oxigenioterapia (BELERENIAN, 2003).

\section{Diagnóstico}

Dentre várias ferramentas utilizadas como requisitos no diagnóstico da TF, devemos sempre levar em consideração em antemão questões relacionadas ao histórico do animal, sinais clínicos e predisposição racial (GOODWIN, 2002).

A elucidação da Tetralogia de Fallot pode ser obtido por exame radiográfico, diante da evidenciação de aumento ventricular direito, dilatação da artéria pulmonar e hipoperfusão pulmonar. Os achados eletrocardiográficos são consistentes com aumento ventricular direito, eixo desviado para direita e onda S profunda. Arritmias são incomuns, mas podem ocorrer (PATTERSON et al., 1974; EYSTER, 1993; LEW et al., 1998; LARSSON et al., 2000; BONAGURA \& LEHMKUHL, 1998; ABBOT, 2000; WARE, 2003).

$\mathrm{Na}$ hematologia é usual encontrar policitemia em cães e gatos com cianose em repouso, devido à TF. No entanto, quanto mais novo o paciente, menos notável é o aumento no hematócrito. Belerenian (2003) observou um felino siamês de 2 meses de idade em repouso, com cianose e um hematócrito de $37 \%$ e um cão Poodle Toy de um ano de idade com o hematócrito de $80 \%$. Isso reflete a variabilidade da resposta dos valores de eritropoietina renal. Se for acessível, a análise de gases sanguíneos é de grande valor. 
MENDES, R.S., SOUZA, A.P. e SANTANA, V.L. Tetralogia de Fallot em pequenos animais Revisão bibliográfica. PUBVET, Londrina, V. 6, N. 3, Ed. 190, Art. 1279, 2012.

A ecocardiografia assume um importante método de diagnóstico para a Tetralogia de Fallot, onde reflete alterações em dimensões de câmaras e presença de defeitos estruturais cardíacos (BONAGURA, 1992; FOSSUM, 1997; TIDHOLM, 1997; LARSSON et al., 2000). As características anatômicas da TF, reconhecíveis pela ecocardiografia, incluem (PATTERSON et al., 1974):

- Defeito do septo interventricular em sua porção membranosa, de tamanho variável, comumente grande e de fácil comunicação (exceto nas casos em que a hipertrofia intensa diminui o orifício interventricular);

- Desalinhamento da direita da inserção da artéria aorta (dextroposição, que pode se localizar a esquerda, à direita ou sobre o defeito do septo interventricular; a aorta também pode apresentar seu diâmetro aumentado;

- Estenosa valvar e/ou subvalvar pulmonar, com obstrução da via de saída do ventrículo direito; hipoplasia da artéria pulmonar secundária a obstrução também pode estar presente;

- Hipertrofia secundária marcante do ventrículo direito (conseqüência da sobrecarga de pressão causada pela estenose pulmonar), do tipo concêntrica.

- Em algumas situações esta hipertrofia é de tal magnitude, que acaba por levar a diminuição do orifício do defeito do septo interventricular. Em outras, a projeção do septo hipertrofiado em via de saída o ventrículo esquerdo leva a ocorrência de obstrução dinâmica. Nestes casos, complica-se a avaliação subjetiva do posicionamento da aorta em relação ao septo interventricular.

A angiografia é outro método diagnóstico de valia revelando hipertrofia ventricular direita, estenose da valva pulmonar, defeito de septo interventricular, cavidade ventriculares com dimensões reduzidas, aorta descendente mais calibrosa e dextroposição da aorta (BONAGURA, 1992; EYSTER, 1993; LEW et al., 1998). A angiografia seletiva do ventrículo direito revela um preenchimento simultâneo das artérias aorta e pulmonar, em 
MENDES, R.S., SOUZA, A.P. e SANTANA, V.L. Tetralogia de Fallot em pequenos animais Revisão bibliográfica. PUBVET, Londrina, V. 6, N. 3, Ed. 190, Art. 1279, 2012.

contrapartida a não seletiva propicia o diagnóstico da TF, mas é uma técnica muito menos sensível que a ecocardiogafia (GOODWIN, 2002).

Deve-se atentar para o diagnóstico diferencial com outras afeç̧ões cardíacas congênitas também causadoras de quadro cianótico, como a estenose da valva pulmonar com defeito de septo interatrial ou interventricular, transposição completa de grandes vasos, persistência de ducto arterioso, atresia pulmonar com septo interventricular íntegro e, mais raramente, atresia da valva pulmonar com intercomunicação atrial, septo interventricular íntegro e uma marcante estenose de tricúspide (ETTINGER \& SUTER, 1970; FOSSUM, 1997, BELERENIAN, 2003).

\section{Tratamento}

A Tetralogia de Fallot, como também constatado em outras moléstias cardíacas congênitas, pode ser tolerada durante anos pelo animal, principalmente se o fluxo sanguíneo pulmonar for mantido e a policitemia secundária for controlada (BONAGURA, 1992).

Alguns gatos podem tolerar este defeito durante anos, mesmo com a ocorrência de hipóxia, policitemia e síndrome de hiperviscosidade (PATTERSON et al., 1874; WARE, 2003).O tratamento pode ser tanto paliativo quanto curativo. O tratamento paliativo pode ter como base a terapia medicamentosa ou a intervenção cirúrgica. Os animais que apresentam saturação de oxigênio arterial menor que $70 \%$ são indicados para serem submetidos à intervenção cirúrgica paliativa (FOSSUM, 1997; EYSTER, 1993; LARSSON et al., 2000).

\section{Tratamento médico}

Em animais com cianose grave e policitemia progressiva, podem realizar-se flebotomias com substituição do sangue extraído por duas vezes o volume de solução cristalóides como Ringer com lactato ou $\mathrm{NaCl}$ 0,9\%. Com o intuito de manter o hematócrito entre 60 e $65 \%$. Diante das complicações 
MENDES, R.S., SOUZA, A.P. e SANTANA, V.L. Tetralogia de Fallot em pequenos animais Revisão bibliográfica. PUBVET, Londrina, V. 6, N. 3, Ed. 190, Art. 1279, 2012.

tromboembólicas decorrentes da policitemia, pode-se usas aspirina em doses baixas (GOODWIN, 2002; BELERENIAN, 2003).

Se flebotomias freqüentes forem requeridas pode se usar hidroxiuréia, já que ela produz mielossupressão reversível. Deve-se começar com a dose de $30 \mathrm{mg} / \mathrm{kg} / \mathrm{dia}$, durante sete a dez dias, em seguida, continuar com $15 \mathrm{mg} / \mathrm{kg} / \mathrm{dia}$. Deve monitorar semanalmente a contagem de glóbulos vermelhos e plaquetas. Os efeitos adversos são vômitos, anorexia, e hipoplasia medular (BELERENIAN, 2003).

Para os casos em que o período de hipóxia foi longo, recomenda-se repouso ou confinamento, oxigenioterapia, administração de sulfato de morfina e bicarbonato de sódio para os animais que apresentem acidose metabólica (BONAGURA, 1992).

Os beta-bloqueadores são utilizados para diminuir a hipercontratilidade do infundíbulo hipertrofiado do VD e atenuar vasodilatação sistêmica mediada pelos receptores $\beta$-adrenérgicos. $O$ aumento da resistência vascular sistêmica reduz a magnitude do "shunt" da direita para esquerda. Tudo isso, melhora e previne as crises hipóxicas, promovendo um aumento no fluxo sanguíneo pela artéria pulmonar, alem de exercer o efeito lusitrópico (GOODWIN, 2002; BELERENIAN, 2003; EYSTER, 1993; LARSSON et al., 2000, PATTERSON et al, 1974; ADAMS, 2003).

\section{Tratamento cirúrgico}

\section{Cirurgia Paliativa}

As técnicas paliativas de correção são as mais indicadas, por sua praticidade e pelos bons resultados. Essa cirurgia consiste em produzir uma anastomose sistêmica pulmonar, para direcionar o fluxo sanguíneo pulmonar, aumentando o retorno do sangue oxigenado ao $V E$ e, conseqüentemente, à circulação sistêmica (BELERENIAN, 2003). 
MENDES, R.S., SOUZA, A.P. e SANTANA, V.L. Tetralogia de Fallot em pequenos animais Revisão bibliográfica. PUBVET, Londrina, V. 6, N. 3, Ed. 190, Art. 1279, 2012.

A terapêutica primária cirúrgica paliativa utilizada consiste em procedimentos de Blalock-Taussig, que consiste numa conexão entre a aorta e a artéria pulmonar utilizando-se a artéria subclávia esquerda como enxerto (Blalock-Taussig), sendo o resultado bastante compensador, minimizando sensivelmente os efeitos da hipóxia (BONAGURA, 1992; EYSTER, 1993; LARSSON et al., 2000). Outras técnicas também podem ser adotadas como: Blalock-Taussig modificada: Blalock-Taussig, com enxerto autólogo de subclávia ou prótese de Goretex; Potts: anastomose da aorta ascendente à artéria pulmonar ou à pulmonar esquerda; Waterson-Cooley: anastomose da aorta à artéria pulmonar direita. Anastomose terminoterminal, entre a artéria torácica interna esquerda e a artéria lobar do lóbulo médio esquerdo (em felinos, com neuromectomia posterior). Atualmente, a técnica de eleição para correção paliativa, é a de Blalock-Taussig modificada (BELERENIAN et al., 2003).

Técnica de Blalock-Taussig

Primariamente, sob condições anestésicas adequadas, baseadas em prémedicação com opióides (morfina ou meperidina), indução por propofol e manutenção com Isofluorano ou Sevofluorano, o animal deve ser colocado em decúbito lateral direito, para uma abordagem lateral esquerda, previamente submetido assepsia em toda a margem cutânea lateral. $04^{\circ}$ espaço intercostal é o acesso de eleição para a toracotomia intercostal padrão em intervenções desta natureza (FANTONI, 2002; IRISAWA et al., 1981; FOSSUM, 1997; BELERENIAN et al., 2003);

Firmado o acesso a cavidade torácica, procede-se a identificação da artéria subclávia esquerda, separada das estruturas do espaço mediastínico após dissecação o mais cranial possível e dorsal a artéria braquiocéfalica. Logo em seguida, realiza-se a abertura do pericárdio na porção dorsal e paralelo ao nervo frênico (FOSSUM, 1997, BELERENIAN et al., 2003). 
MENDES, R.S., SOUZA, A.P. e SANTANA, V.L. Tetralogia de Fallot em pequenos animais Revisão bibliográfica. PUBVET, Londrina, V. 6, N. 3, Ed. 190, Art. 1279, 2012.

Após delimitação da artéria subclávia esquerda, esta por sua vez é ligada na altura da primeira bifurcação por uma pinça cardiovascular atraumática (Pinça Satinski), na emergência com a aorta (Figura 2). Em seguida, é direcionada à artéria pulmonar, ocluída e incisionada, onde será feita a anastomose com fio de polipropileno 7-0 devido ao pequeno calibre da artéria subclávia esquerda (IRISAWA et al., 1981; FOSSUM, 1997). Anastomose finalizada retira-se a pinça Satinski da artéria subclávia esquerda e da artéria pulmonar, para permitir o fluxo sanguíneo.

Uma modificação da técnica de Blalock-Taussig foi desenvolvida, com intuito de minimizar o ângulo na origem da artéria subclávia esquerda, uma vez que, tem-se relatado situações de oclusão até obstrução do vaso, observado geralmente na origem da artéria subclávia esquerda devido a um ângulo de $170^{\circ}$ formado na região (KEAGY et al., 1985; FOSSUM, 1997; IRISAWA et al., 1981).

A técnica de Blalock-Taussig modificada consiste no pinçamento e secção da junção aorta-artéria subclávia esquerda com pinça Satinski, onde logo é reconstruída, de modo a estabelecer um ângulo de $90^{\circ}$ entre os vasos, determinando desta forma, a não oclusão como descrito anteriormente. Daí por diante, segue o mesmo procedimento de anastomose como mencionado para técnica simples (KEAGY et al., 1985; FOSSUM, 1997). O uso de enxerto vascular sintético de politetrafluoretileno já foi descrito como uma variação da técnica, para estabelecer um desvio do fluxo sanguíneo (WEBER et al., 1995). 
MENDES, R.S., SOUZA, A.P. e SANTANA, V.L. Tetralogia de Fallot em pequenos animais Revisão bibliográfica. PUBVET, Londrina, V. 6, N. 3, Ed. 190, Art. 1279, 2012.
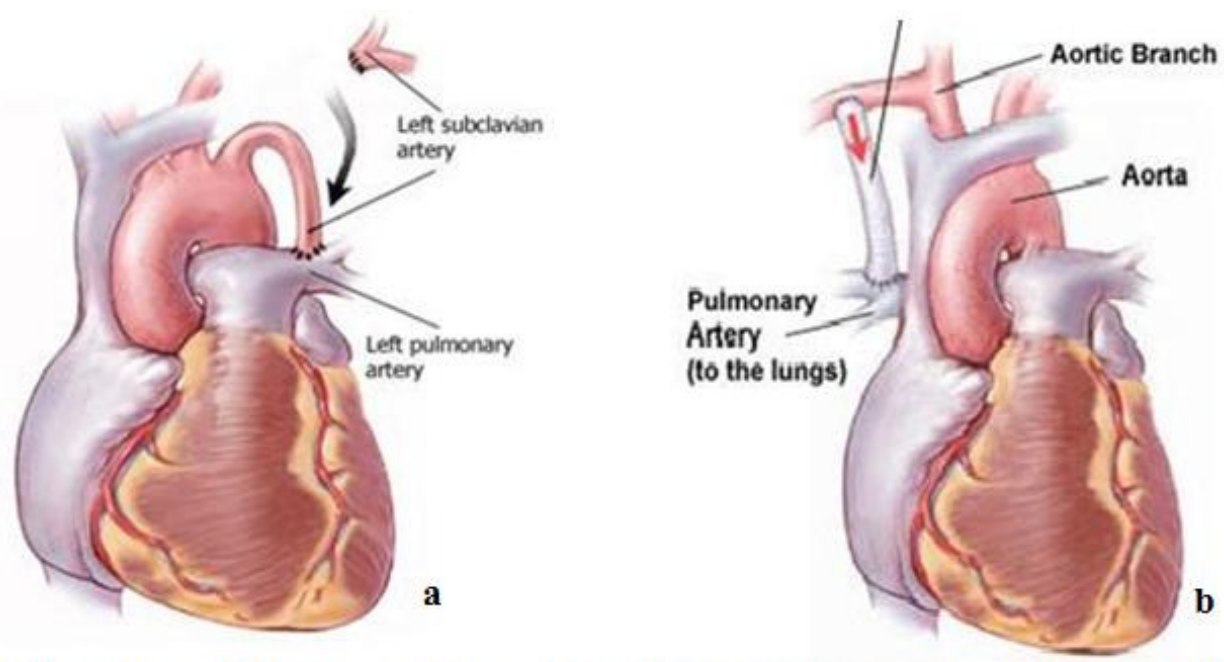

Figura 2: a. Cirurgia paliativa de Blalock-Taussig, demonstrando o shunt entre a aorta e a artéria pulmonar utilizando-se a artéria subclávia esquerda como enxerto (WEBER et al., 1995). b. Cirurgia paliativa de Blalock-Taussig modificada, onde é possível observar demonstrando o shunt entre o ramo da aorta e a artéria pulmonar utilizando-se enxerto sintético (WEBER et al., 1995).

\section{Cirurgia Corretiva}

No tratamento definitivo, é utilizada a circulação extracorpórea, os defeitos septal ventricular e do infundíbulo do VD são corrigidos com enxertos de pericárdio autólogo (ou com um prótese) e plastia da valva e artéria pulmonar. Tal procedimento raramente é feito em medicina veterinária (BONAGURA, 1992; LEW et al., 1998; BELERENIAN, 2003).

\section{Prognóstico}

O procedimento cirúrgico de ordem paliativa para correção da Tetralogia de Fallot revelou-se ser uma satisfatória opção de tratamento, reduzindo significativamente quadros de hipoxemia. Porém não é incomum, mortes súbitas em animais portadores desta cardipatia congênita, principalmente em condições de hipóxia, hiperviscosidade e disritmias cardíacas (BONAGURA, 1992; EYSTER, 1993). Ainda, segundo Eyster (1993), animais portadores, que não sejam submetidos a qualquer terapia de correção, não chegam a uma 
MENDES, R.S., SOUZA, A.P. e SANTANA, V.L. Tetralogia de Fallot em pequenos animais Revisão bibliográfica. PUBVET, Londrina, V. 6, N. 3, Ed. 190, Art. 1279, 2012.

sobrevida de um ano. Enquanto, os que receberam intervenção cirúrgica, possuem como fase crítica o primeiro mês, uma vez superada, apresentam uma sobrevida alta.

Já os animais submetidos ao tratamento cirúrgico corretivo, com intervenção sobre o septo interventricular e na estenose pulmonar, vêm assumindo um caráter de risco sobre sua aplicação nesta cardiopatia congênita, diante dos altos índices de insucesso, atingindo cerca de $75 \%$ de mortalidade (EYSTER, 1993; LARSSON et al., 2000). Os animais portadores de Tetralogia de Fallot, que apresentam estenose pulmonar discreta, sem quadros cianóticos, possuem prognóstico favorável (FOSSUM, 1997).

\section{CONSIDERAÇÕES FINAIS}

A Tetralogia de Fallot é uma importante cardiopatia de ordem congênita, digna de sempre levada em consideração em quadros de síndromes cianóticos na clínica de pequenos animais. Atentando sempre para o estadiamento da enfermidade, a fim de direcionar efetivamente a uma conduta terapêutica cirúrgica paliativa, uma vez que o tratamento médico assume uma grande parcela de insucesso.

A técnica da Blalock Taussig modificada como terapia cirúrgica paliativa é o método recomendado pela maioria dos autores, frente aos altos índices de sobrevivência e baixos de complicações e custos. O mesmo não se atribui ao tratamento cirúrgico definitivo diante dos altos índices de insucessos que gira em torno deste procedimento.

\section{REFERÊNCIAS BIBLIOGRÁFICAS}

ABBOT, J.A. Tetralogy of Fallot. In: Tilley, L.P. \& Smith, F.W.K. The 5-Minute Veterinary Consult. Canine and Feline $2^{\text {nd }}$ ed. Baltimore: Lippincoot Williams \& Wilkins, p.1240-1241. 2000. 
ANDERSON, L. K. Defeitos Cardíacos Congênitos. In: BOJRAB, M. J. Mecanismos da Moléstia na Cirurgia dos Pequenos Animais. São Paulo, Manole, 1996 .p.387-97.

BARTELINGS, M.; GITTENBERGER-de GROOT, A. (1991). "Morphogenetic considerations on congenital malformations of the outflow tract. Part 1: Common arterial trunk and tetralogy of Fallot". Int. J. Cardiol. 32 (2): 213-30.

BELERENIAN, G.C. 2003. Tetralogia de Fallot. In: Belerenian, G.C.; Mucha, C.J; Camacho, A.A. Afecções Cardiovasculares em Pequenos Animais. São Paulo. Ed. Interbook, p. 140-145.

BELERENIAN, G.C.; PUCHETA, C.; BOUQUeT, O.M. 2003. Cirurgia Cardiovascular. In: In: Belerenian, G.C.; Mucha, C.J; Camacho, A.A. Afecções Cardiovasculares em Pequenos Animais. São Paulo. Ed. Interbook, p. 271-282.

BOLTON, G.R.; ETTINGER, S.J.; LIU, S.K. Tetralogy of Fallot in three cats. J Am Vet Med Assoc, v.160, n.12, p.1622-31, 1972.

BONAGURA, J.D. \& LEHMKUHL, L.J. Congenital heart disease. In: Fox P.R., Sisson D., Möise N.S. Textbook of canine and feline cardiology, W.B. Saunders, p.515-519. 1998.

BONAGURA, J.D. Moléstia cardíaca congênita. In: Ettinger, S.J. Tratado de medicina interna veterinária - Moléstias do cão e do gato. São Paulo : Manole, 1992. p.1026-82.

BOON, J.A. Congenital heart disease In: Boon, J.A. Manual of veterinary echocardiography. Baltimore: Williams \& Wilkins, p.431-435. 1998.

ETTINGER, S.J.; SUTER, P.F. Congenital heart disease. In: ETTINGER, S.J.; SUTER, P.F. Canine cardiology. Philadelphia :Saunders, 1970. p.497-602.

EYSTER, G.E. Basic cardiac surgical procedures. In: Slatter, D. Textbook of small animal surgery. 2. ed. Philadelphia :Saunders, 1993. V.1, p.893-918.

FANTONI, D.T. Anestesia no cardiopata. In: Fantoni, D.T.; Cortopassi, S.R.G. Anestesia em cães e gatos. São Paulo : ROCA, 2002. p.294-320.

FOSSUM, T.W. Surgery of the cardiovascular system. In: FOSSUM, T.W. Small animal surgery. Missouri : Mosby-Year Book, 1997. p.575-608.

GOODWIN, J.K. 2002. Cardiopatias Congênitas. In: Tilley, L.P. \& Goodwin, J.K. Manual de Cardiologia. Terceira edição. São Paulo: Ed. Roca, p. 259 -276.

IRISAWA, T. et al. The clinical and experimental studies on the hemodynamic influence of the shunt flow through anastomosis of Blalock-Taussig operation. Nippon Kyobu Geka Gakkai Zasshi, v.29, n.9, p.1409-1416, 1981.

KEAGY, B.A. et al. Changes in ventricular hemodynamics caused by a systemic-pulmonary shunt. Journal Surg Res, v.39, v.4, p.294-299, 1985.

KITTLERSON, M.D. Tetralogy of Fallot. In: Kittlerson, M.D. \& Kienle, R.D. Small Animal Cardiovascular Medicine. St. Louis: CV Mosby, p.240-247. 1998.

KOBAYASHI, J.; KAWASHIMA, Y.; MASUDA, H. et al: Prevalence and risk factors of tricuspid regurgitation after correction of tetralogy of Fallot. J Thorac Cardiovasc Surg 1991; 102: 611-16. 
LARSSON, M.H.M.A. et al. Clinical diagnosis and alternative surgical treatment of tetralogy of Fallot in a dog. A case report. Arquivo Brasileiro de Medicina Veterinária e Zootecnia, v.52, n.5, p.433-436, 2000.

LEW, L.J. et al. Open-heart correction of tetralogy of Fallot in an acyanotic dog. J Am Vet Med Assoc, v.213, n.5, p.652-657, 1998.

MILLER, M. W; BONAGURA, J. D. Cardiopatia Congênita. In: BIRCHARD, S. J; SHERDING, G. S. Manual Saunders Clínica de Pequenos Animais. São Paulo, Roca, 1998. p. 564-69.

PATTERSON, D.F., et al. A single gene defect underlying conotruncal malformations interferes with myocardial growth during embryonic development: Studies in the CTD line of Keeshound dogs. Am J Hum cardiology 34:388. 1993.

PATTERSON, D.F.; PYLE, R.L.; VAN MIEROP, L. et al. Hereditary defects of the conotruncal septum in Keeshound: Pathologic and genetics studies. Am J Cardiology 34:187. 1974.

SISSON, D.D.; THOMAS, W.P.; BONAGURA, J.D. In: Ettinger. Textbook of Veterinary Internal Medicine. Diseases of the Dog and Cat. $5^{\text {a }}$ ed. W.B. Saunders Company. 1992.

TIDHOLM, A. Retrospective study of congenital heart defects in 151 dogs. Journal Small Animal Practice, v.38, n.3, p.94-98, 1997.

WARE, W.A. Congenital cardiovascular diseases. In: Ware, W.A. Cardiovascular disease in small animal medicine. London: Copyright Manson Publishing, p.228-262. 2007.

WARE, W.A. Common congenital cardiac anomalies. In: Nelson, R.W \& Couto, C.G. Small Animal Cardiovascular Medicine. St. Louis. Mosby, 151-168. 2003.

WEBER, U.T. et al. Palliative treatment of tetralogy of Fallot using a PTFE (polytetrafluoroethylene) vascular graft. Schweiz Arch Tierheilkd, v.137, n.10, p.480-484, 1995. 